\title{
Size Effects: Moving Forwards
}

\author{
X. Hu and K. Duan \\ Department of Mechanical Engineering, University of Western Australia \\ 35 Stirling Hwy, Crawly, Western Australia 6009 \\ xhu@mech.uwa.edu.au
}

\begin{abstract}
A common size effect mechanism exists in fracture of vastly different materials with huge differences in size. The common mechanism is governed by the interaction of the critical crack, surface condition and material microstructures. The distance of a crack tip to the surface thus becomes an important measurement, which can either be the crack size itself or uncracked ligament depending on whether a shallow or deep crack is considered. The surface roughness and surface flaws form an effective boundary layer in micro-specimens/structures such as thin films and MEMS structures regardless whether they are amorphous, or have extremely fine micro-structures. The boundary layer in macro-specimens such as polycrystalline ceramics and concrete is determined by the material micro-structures and fracture process zone, which is also determined by the material micro-structure. The common size effect mechanism is then linked to the thickness or depth of the boundary layer and its ratio to the crack length or uncracked ligament.
\end{abstract}

\section{Introduction}

The phenomenon of size effects on fracture of brittle and quasi-brittle materials is no longer confined to macro-specimens and structures measured from millimetres to meters because of the evolution of nano-technology, micro-machines and devices. For instance, strengths of thin films and similar MEMS (micro-electro-mechanical systems) structures measured from a few hundred nano-meters (nm) to a few microns $(\mu \mathrm{m})$ in thickness show size effects regardless whether the micro-specimens used to measure the strengths are single crystals, amorphous or have extremely fine micro-structures [1-4]. The surface defects responsible for fracture of those micro-specimens can vary from $\mathrm{nm}$ to $\mu \mathrm{m}$ depending on how the specimens have been prepared. Despite of the huge difference in flaw and specimen size between the aforementioned micro- and macro- specimens, and their vastly different microstructures, a common feature can still be found in terms of fundamental size effect mechanisms.

The objective of this paper is to show such a common mechanism exists through discussions of size effects of very different materials with specimen size varying from a few hundred nm to meters.

\section{Size Effects on Fracture of Single Crystal Silicon}

The Weibull strengths of single crystal silicon by micro- bending tests [1] are shown in Fig. 1. Details on the micro-specimens measured in $\mu \mathrm{m}$ and sub- $\mu \mathrm{m}$ (a few hundreds $\mathrm{nm}$ ) are listed in Table 1. It is clear that both the strength and Weibull modulus (or the slope of the strength distribution) vary with the specimen size. Although the strength of a brittle solid is well known to be size dependent, the physical meaning of the Weibull modulus variation with the specimen size is much less well understood, and therefore deserves more attention. 
The smallest nano-flaw specimens yield the largest Weibull modulus, and the highest strength, which is fairly close to the theoretical strength of silicon.

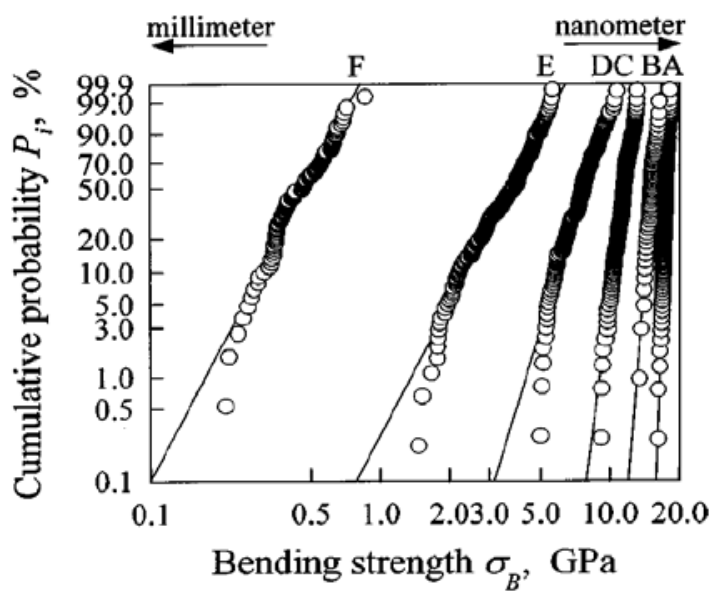

Figure 1: Weibull strength distributions showing size effect on strength and Weibull modulus [1]

The specimen details and Weibull parameters are listed in Table 1. Two different manufacturing techniques were used to fabricate those specimens [1]. The field-enhanced anodization was used to fabricate the nano-flaw specimens, Group A, B and C, and the conventional photolithography technique was used to fabricate micro-flaw specimens, Group D, E and F.

Table 1. Weibull parameters, and specimen details

\begin{tabular}{|c|c|c|c|c|c|c|}
\hline & \multicolumn{3}{|c|}{ nano-flaw specimens } & \multicolumn{3}{c|}{ micro-flaw specimens } \\
\hline Group & A & B & C & D & E & F \\
\hline$L(\mu \mathrm{m})$ & 6 & 6 & 6 & 35 & 360 & 9,850 \\
\hline$t(\mu \mathrm{m})$ & 0.255 & 0.255 & 0.255 & 1.91 & 19 & 520 \\
\hline$w_{l}$ & 0.2 & 0.3 & 0.8 & 4.75 & 48 & 1,045 \\
\hline$w_{2}$ & 0.37 & 0.47 & 0.98 & 7.5 & 74.5 & 1800 \\
\hline$\sigma_{*}(\boldsymbol{G P a})$ & $\mathbf{1 7 . 6 4}$ & $\mathbf{1 5 . 4 2}$ & $\mathbf{1 1 . 7 6}$ & $\mathbf{8 . 0 9}$ & $\mathbf{4 . 1 4}$ & $\mathbf{0 . 5 3}$ \\
\hline $\boldsymbol{M}$ & $\mathbf{6 2 . 0 5}$ & $\mathbf{2 6 . 6 2}$ & $\mathbf{1 6 . 8 1}$ & $\mathbf{7 . 2 4}$ & $\mathbf{4 . 2 0}$ & $\mathbf{4 . 1 8}$ \\
\hline$L \cdot w_{l}\left(\mu m^{2}\right)$ & 1.2 & 1.8 & 4.8 & 166 & $1.7 \cdot 10^{4}$ & $1.0 \cdot 10^{7}$ \\
\hline
\end{tabular}

In the case of single crystal silicon, the concept of fracture toughness as an energy criterion should be applicable even at the atomic scale if a crack is atomically sharp. Therefore, strength predictions from the fracture toughness are influenced only by the interaction of surface defects or between a critical surface defect and surface roughness. If the size effects shown in Fig. 1 among nano-flaw specimens, Groups $\mathrm{A}, \mathrm{B}$ and $\mathrm{C}$ need to be modelled in details, the interaction between the nano-flaws or a critical nano-flaw and surface roughness needs to be modelled rather than the absolute size of the specimens. The small reduction in the width measurements, $\mathrm{w}_{1}$ and $\mathrm{w}_{2}$, and the huge change in Weibull modulus can only be explained by the size reduction and removal of surface defects. An equivalent sharp crack or an equivalent fracture process zone (FPZ) can be assumed to deal with the potential problem on crack-tip sharpness. 
Ignoring the interactions between the nano-flaws, and assuming those nano-flaws are atomically sharp, nano- and micro-flaw distributions and densities have been obtained using $\mathrm{K}_{\mathrm{IC}}=1.0 \mathrm{MPa} \sqrt{\mathrm{m}}$, and shown in Fig. 2 [4]. The number of flaws, $\mathrm{N}$, larger than a, on the tensile surface is shown in the figure. The flaw density can then be calculated using the specimen dimensions listed in Table 1 . The flaw statistics [4] indicates that the size effects shown in Fig. 1 are far more complicated than a simple strength variation with the specimen size. The difference in the surface flaw density is at least another factor to be considered. The flaw statistics [4] also shows that Weibull modulus is directly related to the surface flaw distribution. The slops of flaw distribution curves in Fig. 2 are determined by Weibull modulus. Therefore, the difference in Weibull modulus means specimens of different groups have different surface flaw distributions and surface roughness. The differences in surface flaw density and distribution and the closely related surface roughness show that modelling of the boundary layer is essential to the understanding of the size effects. The size effects of polysilicon with an average grain size of around $200 \mathrm{~nm}$ show similar problems [2-4]. The difference in the surface roughness and surface flaw of micro-beam specimens again dominates the size effects. As a result, the boundary layer again should be the focus of modelling.

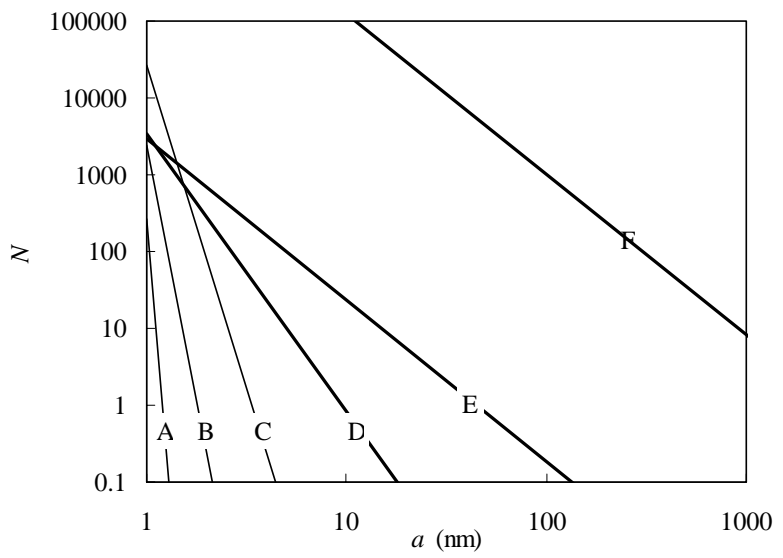

Figure 2: Flaw distributions and number of flaws over the tensile surface based on Weibull strength distributions in Fig. 1 [4]

\section{Size Effects on Fracture of Concrete}

Concrete cannot be more different to the micro-specimens of single crystal silicon and polysilicon discussed in the previous section. A typical concrete structure (e.g. aggregates) is measured at least in $\mathrm{mm}$, and a typical crack size is measured in $\mathrm{cm}$, while testing specimens can be as large as $2 \mathrm{~m}$. Is there anything in common in the size effects on fracture of the micro- single crystal silicon and polysilicon specimens and macro-concrete specimens? It can be shown that from the mechanics viewpoint a common mechanism exists despite of the huge differences in the material micro-structures, specimen size and critical flaw.

In recent years, we have shown that the size effects on quasi-brittle fracture of concrete can be adequately modelled by considering the influence of a boundary layer in a concrete specimen [5-10]. The boundary layer is characterized by its fracture process zone (FPZ), which is related to the concrete structure or aggregate size. Size effects on the specific fracture energy $\mathrm{G}_{\mathrm{F}}$ and the nominal strength $\sigma_{\mathrm{N}}$ have been modelled by considering the influence of the boundary layer, and confirmed by independent experimental results. It shows that similar to fracture of micro-specimens of MEMS materials, the condition of a boundary layer is still the dominant influence on the size effects of macro-specimens such as concrete beams. 
Modelling of the boundary effect or the boundary effect model is based on consideration of the interaction of a FPZ and a free specimen boundary as shown in Fig. 3.

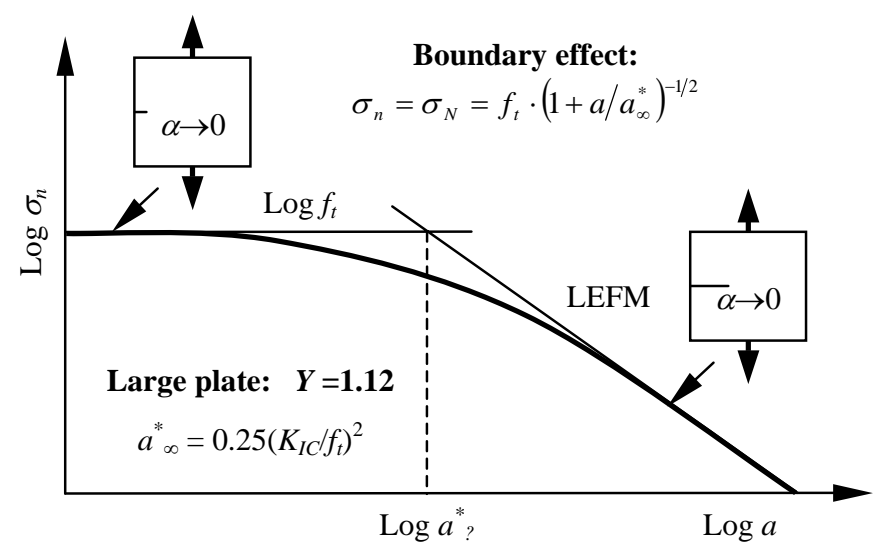

(a)

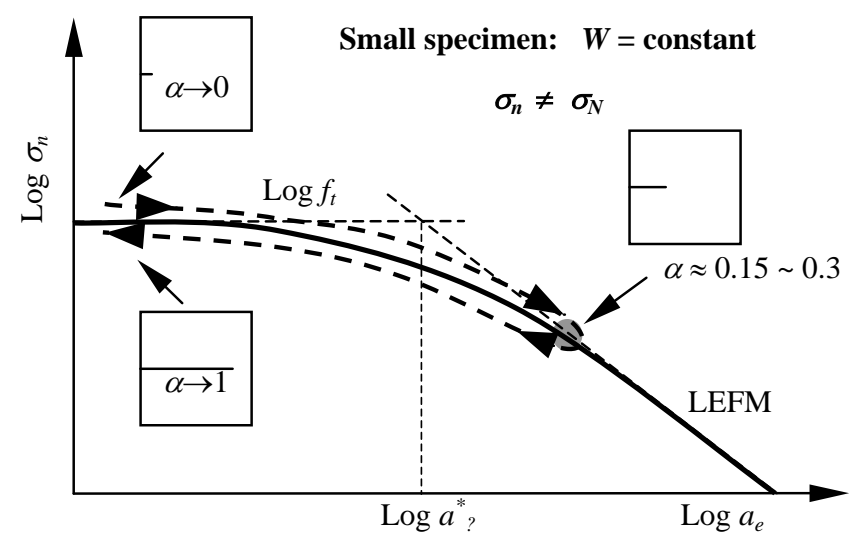

(b)

Figure 3: Influence of the specimen boundary on quasi-brittle fracture of concrete

The crack-tip FPZ is not shown in Fig. 3 for clarity. However, it is known that FPZ can be quite large for concrete-like materials [11-12]. In Fig. 3 (a), the quasi-brittle fracture transition of a very large concrete specimen is induced purely by the interaction between FPZ and the specimen boundary where the edge crack has originated. Fracture modelling of such a large plate does not involve the specimen size. In fact, only the reference crack $a_{\infty}^{*}$, which is proportional to FPZ, is required. Let $\sigma_{N}$ be the nominal strength ignoring the presence of the edge crack, and $\sigma_{\mathrm{n}}$ be the nominal strength considering the presence of the edge crack. Since the crack size a is much smaller than the specimen size $\mathrm{W}$, or $\alpha=\mathrm{a} / \mathrm{W} \rightarrow 0$, the two nominal strengths are identical and is given by: 


$$
\sigma_{N}=\sigma_{n}=\frac{f_{t}}{\sqrt{1+\frac{a}{a_{\infty}^{*}}}}
$$

where $f_{t}$ is the tensile strength and the reference crack a ${ }_{\infty}{ }_{\infty}$ is defined by the intersection of the two fracture criteria, $f_{t}$ and $K_{I C}$. As shown in Fig. 3, this reference crack has also defined a boundary layer, which is comparable to the size of FPZ. Within the boundary layer, the strength criterion is dominant. Away from the boundary layer, concrete can be treated as brittle homogenous material and the fracture toughness criterion $\mathrm{K}_{\mathrm{IC}}$ applies. In this case, the crack a is also the distance of the crack tip to the front boundary. Therefore, the crack ratio $\mathrm{a} / \mathrm{a}_{\infty}{ }_{\infty}$ indicates the relative distance of a crack tip away from the boundary. Clearly, the transition curve given by equation (1), which connects two straight lines in Fig. 3 (a), is not linked to the size variation. However, the same quasi-brittle fracture curve given by equation (1) also defines the fracture transition of small concrete specimens associated with the common size effects. In this case, the nominal strength considering the presence of the edge crack should be used, and is given by:

$$
\sigma_{n}=\frac{f_{t}}{\sqrt{1+\frac{a_{e}}{a_{\infty}^{*}}}}
$$

The equivalent crack $\mathrm{a}_{\mathrm{e}}$ is related to the crack length $\mathrm{a}$, the geometry factor $\mathrm{Y}$ used in the stress intensity formula and the relation between the nominal strengths [5,7-10]. Its function is to determine the dominant influence of boundaries. Different to the large plate situation shown in Fig. 3(a), the shortest distance of a crack-tip to a boundary in the case of a finite-sized specimen can either be the crack length itself or the remaining ligament as shown in Fig. 3(b). The equivalent crack $a_{e}$ is introduced to consider the influence of a nearest boundary. As shown in Fig. 3(b), if the crack a is too deep, the equivalent crack $a_{e}$ is actually reduced so that the remaining ligament is compared with the reference crack $\mathrm{a}_{\infty}{ }_{\infty}$. The important feature is that the quasi-brittle fracture curve of a finite specimen shown in Fig. 3(b) follows exactly the quasi-brittle fracture curve of a very large specimen in Fig. 3(a) determined solely by the boundary influence. The specimen size $\mathrm{W}$ only determines the turning point indicated in Fig. 3(b). When different finite-sized specimens are used in experiment, different turning points lead to the apparent size effects. However, the boundary-effect curve shown in Fig. 3(a) is more fundamental, which shows the true mechanism commonly existing in vastly different materials such as single crystal silicon and concrete discussed in this study. Due to the page limit, experimental verification of the boundary effect model, equations (1) and (2), is not shown, but can be found in refs [5,7-10].

\section{Discussion and Concluding Remarks}

The boundary features of different materials discussed in this study have been summarised in Fig. 4. Surface roughness or various surface defects can form an effective boundary layer in single crystal silicon, which controls the strength of silicon. Similar feature has been identified on surfaces of amorphous silicon nitride thin films, where surface roughness of around $10 \mathrm{~nm}$ controls the strength [13]. Interaction of a critical surface flaw with the boundary layer needs to be considered for both single crystal silicon and amorphous silicon nitride, e.g. in terms of surface roughness. If a critical surface flaw can be identified, equation (1) or (2), may be extended to include the surface roughness measurement although fracture of micro-specimens has the statistical nature due to many surface flaws are involved. For polysilicon, the grain size (around $200 \mathrm{~nm}$ ) has to be considered besides the surface roughness. Therefore, the boundary layer would be much thicker than that of single crystal silicon. For concrete, surface roughness is no longer a concern because of the very coarse material structure. In this case, the boundary layer is determined only by FPZ. For a typical polycrystalline ceramic with an average grain size between 1 to $10 \mu \mathrm{m}$, surface finish is still important because of relatively small FPZ, which is similar to the case of polysilicon.

The boundary effect model, equation (1) or (2), shows that the influence of a boundary layer, critical to fracture of micro-specimens of MEMS materials, is also essential to fracture of macro-specimens of very different materials, concrete and polycrystalline ceramics. This common mechanism on size effects provides a new opportunity for further development of size effect models. 
Surface roughness forms an effective boundary layer

Single crystal silicon boundary layer, proportional to surface roughness, is measured in $\mathrm{nm}$

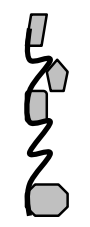

Polysilicon boundary layer, proportional to grain size and surface roughness, is measured in 100 's nm

Figure 4: Boundary layers of different materials should be emphasized instead of specimen size

\section{Acknowledgements}

The authors would like to acknowledge the financial support from the Australian Research Council.

\section{References}

1. $\quad$ Namazu, T., Isono, Y. and Tanaka, T., J. Microelectromechanical Systems, vol. 9, 450-459, 2000.

2. $\quad$ Ding, J.N., Meng, Y.G. and Wen, S.Z., J. Mater. Res., vol. 16, 2223-2228, 2001.

3. $\quad$ Ding, J.N., Yang, Y.C., Cai, L. and Wen, Inter. J. of Nonlinear Sci. and Numerical Simulation, vol. 3, 499-502, 2002.

4. Hu, X.Z. and Duan, K., J. Mater. Sci. and Tech., vol. 12, Suppl. 1, vol. 47, 47-50, 2005.

5. $\quad$ Duan, K., Hu, X.Z. and Wittmann, F.H., Eng. Fract. Mech., vol. 70, 2257-2268, 2003.

6. $\quad$ Hu, X.Z. and Duan, K., Cement and Concrete Res., vol. 34, 1321-1330, 2004.

7. $\quad$ Duan, K. and Hu, X.Z., Strength, Fracture and Complexity, vol. 2, 47-68, 2004.

8. $\quad$ Duan, K., Hu, X.Z. and Wittmann, F.H., Mech. of Mater., 38, 128-141, 2006.

9. Duan, K. and Hu, X.Z., In Fracture Mechanics of Concrete Structures (Proceedings of Framcos-5, April 12-16, 2004, Colorado, USA), ed: by V.C. Li et al., 2004, vol 1, 197-204.

10. Duan, K., Hu, X.Z. and Wittmann, F.H., In Fracture Mechanics of Concrete Structures (Proceedings of Framcos-5, April 1216, 2004, Colorado, USA), ed: by V.C. Li et al., 2004, vol 1, 205-212.

11. Hu, X.Z. and Wittmann, F.H., J. of Mater. in Civil Eng, 2, 15-23, 1990.

12. Hu, X.Z. and Wittmann, F.H., Mater. Struct., 25, 319-326, 1992.

13. Huang, H, Hu, X.Z., Liu, Y., Bush, M, Winchester, K., Musca, C., Dell, J. and Faranone, L., J. Mater. Sci. Tech., 21, 13-16, 2005. 